von denen sich diese Sinneszellen nur durch ihre gestrecktere Gestalt, durch den Besitz eines GeiBelhares am peripheren Ende sowie dadurch unterscheiden, daB sie an ihrer Basis mit Nervenfibrillen in Verbindung stehen. Merkmale, die auf eine bestimmte Sininesfunktion hinweisen, fehlen. In besonders großer Zahl finden sich solche indifferenten Sinneszellen am Schirmrande, wo sie ganze ;Sinnesepithelien“ bilden "könn'en. Auch die Tentakel scheinen reich mit Sinneszellen versehen zu sein.

Es ist sehr wahrscheinlich, daß viele der „indifferenten" Sinneszellen auf Tastreize reagieren. Das kann ails sicher gelten für Sinneszellen, die mit langen steifen Borsten versehen sind, die über die Oberfläche hervorragen. Die Verteilung dieser Tastapparate ist eine ähnliche wie die der indifferenten Sinneszellen; auch sie können in großer Zahl aneinandergereiht sein, so daß „Tastkämme" zustande kommen.

Die Lichtsinnesorgane der Medusen, die „Ocellen" oder "Augenflecke", sind im einfachsten Falle kleine Gruppen von Sinneszellen, die von einer Pigmentmasse eingehüllt werden. Die höher entwickelten Ocellen besitzen außerdem noch kleine lichtbrechende Körper, einfache Linsen, die aus einer Verdickung der Cuticula des Epithels entstehen.

In morphologischer wio physiologischer Hinsicht am interessantestem sind die sagenannten Gehörorgane, die statischen Organe, denen Hertwig seine besondere Aufmerksamkeit zuwandte. In den verschiedenen Abteilungen der Medusen zeigen sie einen sehr verschiedenen $\mathrm{Bau}$, und auch in den einzelnen Abteilungen finden wir eine große Mannigfaltigkeit. Offenbar haben sich diese Organe mehrfach unabhängig voneinander und nach gan $\vec{z}$ verschiedenem Typus entwickelt. Die Gehörorgane des einfachsten Typus bauen sich ausschließlich aus in verschiedener Weise umgebildeten Ektodiermzellen auf. Einzeline Ektodermzellen haben sich in große, derbwandige, mit Flüssigkeit gefüllte Blasen umgewandelt, in deren Mitte sich ein Konkrement befindet. Jede solche Blase wird umgeben von einer wechselnden Zahl ebenfalls modifizierter Ektodermzellen, den „Ḧ̈rzellen", die einerseits mit Nervenfibrillen in Verbindung stehen und andererseits vermittels kurzer, dicker Haare, der „Hörhaare", die Konkrementbliase tragen. Je nachdem ob die Konkrement- und Hörzellen frei an der Oberfläche liegen oder, von der Außenwelt abgeschlossen, in die Tiefe verlagert sind, spricht man von freien oder geschlossenen $\Lambda$ pparaten. Gianz anders gebaut sind die Gehörorgane des zweiten Typus. Sie stellen umgewandelte Tentakel dar, an ihrer $\mathrm{Zu}$ sammensetzung beteiligen sich Ektoderm- und Entodermzellen. In den verschiedenen Gruppen mit tentakulären Gehörorganen läBt sich eine zunehmende Differenzierung dieser Apparate feststellen. Die primitivsten Organe liegen an der Stelle von Tentakeln frei am Schirmrande. Die Basis bildèt das „Hörpolster“, eine hügelig vorgewölbte
Partio des Sinnesepithels, aus stark verlängerten Zellen bestehend, die mit Hörhaaren versehen und an der Basis mit Fibrillen des oberen Nervenrings verbumiden sind. Die Mitte des Hörpolsters trägt den umgewandelten Tentakel; das „Hörkölbchen". Es ist an dem Polster vermiltels eines feinen Stielchens, wio der Klöppel einer Glocke, leicht beweglich befestigt, hängt frei ins Wasser, wird aber von Hörhaaren rings umschlossen. Entsprechend dem Tentakel, aus d'em es hervorgegangen ist, ist es aus einem entodermalen Achsenteil und aus einer einfachen epithelialen Hüllschicht zusammengesetzt. Die terminale Achsenzélle scheidet ein Konkrement aus, das als "Otolith" bezeichnet wird. Wie bei den epithelialen Gehörorganen findet auch bei den tentakulären bei fortschreitender Differenzierung eine Verlagerung in die Tiefe statt, so daß wir auch hier freie und geschlossen $\theta$ Apparate unterscheiden können.

In der zweiten Arbeit haben Richard Hertwig und sein Bruder auch die übrigen Teile des Medusenorganismus einer eingehenden Betrachtung unterzogen (1878). Zwar sind seither die Medusen von vielen Seiten neu untersucht worden, vermittels einer verfeinerten Technik ist es auch vielfach gelungen, noch tiefer in den Bau der einzelnen Organsysteme einzudringen, durch Auffindung neuer, abweichender Formen ließen sich unsere Kenntnisse verbreitern. Im wesentlichen aber brachten die neuen Untersuchungen doch nur eine Bestätigung der Hertwigschen Befunde, und man darf wohl behaupten, daß es nur wenigen Forscherm gelungen ist, mit einem Male so viek Licht über die Organisation einer Tiergruppe zu verbreiten, wie Richard Hertwig und seinem Bruder mit den Untersuchungen über den Bau der Medusen.

\section{Richard Hertwig und der biologische Unterricht an den höheren Lehr- anstalten.}

Von C. Zimmer, München.

Dem gewaltigen Aufschwung, den die biologischen Naturwissenschaften im letzten halben Jahrhundert genommen haben, entsprach und entspricht nicht ihre Stellung im Unterrichte an den höheren Lehranstalten. Diese zu erringen ist Kampf nötig, und unter den Vorkämpfern steht auch Richard Hertwig.

Auf der Hamburger Versammlung deutscher Naturforscher und Arzte im Jahre 1901 hielt Ahlborn in einer stark besuchten gemeinsamen Sitzung der biologischen Sektionen einen Vortrag über die gegenwärtige Lage des biologischen Unterrichts an den höheren Schulen, an dessen Schlub or eine Anzahl von Thesen aufstellte. In der lebhaften Diskussion nahm auch Hertwig das Wort, um aus seinen Erfahrungen als Examinator in der Lehramtsprüfung zu 
sprechen:- Da der künftige Lehrer der Naturwissenschaften unter den bestehenden Verhältnissen niemals Gelegenheit hat, biologischen Unterricht in höheren Klassen zu erteilen', wirft er sein ganzes Interesse im Studium auf die exakten Naturwissenschaften und beschränkt sich in der Biologie auf đas unumgänglich notwendige. Die Nachfrage nach biologischen Spezialvorlesungen auf den Universitäten ist demgemäB nur gering, ein Angebot lohnte sich somit nicht. Den Forderungen auf Schaffung neuer Professuren wurde nicht entsprochen, ja bestehende Professuren wurden eingezogen. So leidet der an und für sich schon unzureichende biologische Unterricht noch dadurch, daB er von Kräften erteilt wird, die dafür teils ein nur mangelhaftes Interesse, teils eine ungenügende Ausbildung besitzen. Eine Änderung verspricht sich Hertwig nur bei einer größeren Berücksichtigung der Biologie im Lehrplan der höheren Schulen.

Zur Ausarbeitung und Verbreitung der Thesen wurde ein Komitee gewählt, dem auch Hertwig angehörte. Der Erfolg war ungeheuer, der Widerhall, den die Thesen fanden, ungeahnt. Für die Breslauer Versammlung (1904) der Naturforscher und Ärzte war eine eingehende Besprechung der Frage auf die Tagesordnung gesetzt. Zur Vorbereitung gab Max Verworn ein Schriftchen heraus, das Wünsche und Vorschläge aus Hochschullehrerkreisen enthielt. (Beiträge zur Frage des naturwissenschaftlichen Unterrichtes an den höheren Schulen, Jena 1904.) Hertwig lieferte hierzu einen Beitrag über die „Erfordernisse der Vorbildung der Mittelschulen für das Studium der Zoologie". Eine Reihe von Gedanken, die er in diesem Rahmen nur streifen konnte, führte or in einem Aufsatz ,Zur Frage der Organisation des zoologischen Unterrichts an den höheren Schulen" aus, der in "Natur und Schule", Band 3, Heft 11, 1904, erschien.

Die Breslauer Tagung setzte eine Kommission zur Verbesserung des naturwissenschaftlichen und mathematischen Unterrichtes ein. Ihre Vorschläge wurden von der Meraner (1905) und Stuttgarter (1906) Versammlung genehmigt. Nach $\mathrm{Ab}$ schluB der Tätigkeit löste sich die Kommission in Dresden (1907) auf und machte einem ,Ausschuß zur Förderung des mathematischen und naturwissenschaftlichen. Unterrichtes" Platz, der aus Vertretern der großen naturwissenschaftlichen und mathematischen Vereine und Gesellschaften gebildet war. Die Deutsche Zoologische Gesellschaft sandte Richard Hertwig und Karl Kraepelin ab.

Auf die Aus- und Vorbildung der biologisehen Lehrkräfte, die ja untrennbar mit der Frage des Unterrichtes verbunden ist, konnte Hertwig in Bayern maßgebenden Einfluß ausüben: Der Kommission zur Vorbereitung der 1912 erfolgten Neuregelung der „Prüfungsordnung für das Lehramt an den höheren Lehranstalten in Bayern" gehörte er als Mitglied und Fachvertreter der Zoologie an.

Skizzieren wir den Standpunkt, den Hert- wig einnimmt an der Hand der Gedankengänge in seinen beiden genannten Schriften:

Der biologische Unterricht in den neunklassigen Anstalten soll sich durch die unteren vier Klassen in je zwei Wochenstunden auf Systematik und Biologie erstrecken. Nach einer. Unterbrechung in zwei Mittelklassen erfolge dann in den drei höchsten Klassen in je zwei Wochenstunden ein anatomisch-physiologischer Unterricht.

Die Zweiteilung des Unterrichts ist nicht nur in mancher anderen Beziehung praktisch, sondern entspricht auch dem doppelten $Z$ wecke, den die Schule zu verfolgen hat:

Einmal soll sie die im Schüler schlummernden Anlagen und Fähigkeiten allseitig zur Entfaltung bringen, so auch die Fähigkeit zu beobachten und das Beobachtete in verständlicher Form wiederzugeben. Bei der bisher überwiegenden, an den humanistischen Anstalten fast ausschlieBlichen Betonung des sprachlich-historischen Bildungsstoffes werden die genannten Fähigkeiten nur ungenügend ausgebildet, ja sogar geradezu zur Verkümmerung gebracht. Hier ein Gegengewicht zu bilden, ist gerade der naturwissenschaftliche Unterricht berufen, insonderheit der biologische; denn er ist mit seinen nach einer komplizierteren Gesetzmäßigkeit verlaufenden Vorgängen darin den exakten Naturwissenschaften weit überlegen, die es mit verhältnismäßig ein. fachen und leicht zu überblickenden Erscheinungen zu tun haben. Als angenehme und sehr erstrebenswerte Beigabe mag betrachtet werden, daß dio biologischen Fächer den Kulturmenschen wieder der Natur, der er entfremdet wurde, nahebringen und geeignet sind, Lièbe $\mathrm{zu}$ ihr zu erwecken und die in der Beschäftigung mit ihr enthailtenen ethischen Bildungsstoffe zugänglich zu machen.

Diese eine Aufgabe der Schule, die Entwicklung der Fähigkeiten, zu erfüllen, ist vor allem Sache des systematisch-biologischen Unterrichts in den unteren Kilassen. Unter Befolgung der induktiven Methode soll mit Betrachtung einzelner biologisch interessanter Typen. der Wirbeltiere, zunächst der Säugetiere begonnen werden. Beschreibung des Habitus, der systematisch wichtigsten anatomischen Merkmale; Schilderung der Lebensweise sollen in einer elementaren, keine hohen Ansprüche an das Gedächtnis stellenden Form gegeben werden. Weitere Vertrieter der Klasse aus anderen Familien und dann Ordnungen werden behandelt, und so das an ider einzelnen Form Gelernte verallgemeinert. Endlich wird als AbschluB der Wirbelitiere die Charakteristik des Stammes herausgearbeitet. In ähnlicher Weise folgen die Arthropoden, die Mollusken, unter besonderer. Berücksichtigung der Schnecken und Muscheln und endlich die Würmer. Letztere werden schon, wie erst recht die Echinodermen, Coelenteraten und Protozoen nur noch kursorisch behandelt. Die gewaltige Fülle des Stoffes zwingt dazu, den ganzen Unterricht in den un- 
terem Klassen frei von Pedanterie und Schablone zu gestalten und dem Iehrer viel freie Auswahl zu. lassen, dio er nach eigenen Neigungen und unter Berücksichtigung der Neigung der Schüler betätigen mag.

Die zweite Aufgabe der Schule ist es, dem Schüler die Kenntnisse und alligemeinen Vorstellungen zu bieten; die er einmal nötig hat, um den großen Fragen im geistigen Leben der Zeit nicht verständnislos gegenüberzustehen. Vorstellungen vom Leben, von Fortpflanzung und Vererbung, von der Stellung des Menschen zur organischen Natur, alles das sind Fragen, die das geistige Leben unserer Zeit tief bewegen. Dem Schüler auf diesem Gebiete die erforderlichen Kenntnisse und Vorstellungen zu vermitteln, ist Sache des vergleichend-aniatomischphysiolagischen Unterrichts in den höheren Klassen. Er beginnt mit der Betrachtung der Zelle, die zur Besprechung der einzelligen Or. ganismen aus dem Tierreiche sowohl wie aus dem Pflanzenreiche, und des Gemeinsamen und Trennenden der beiden organischen Reiche führt. Es folgt die Behandlung der vielzelligen Organismen unter stetem Hinweis darauf, wie durch Arbeitsteilung die Vervollkommnung des Bauplanes, die Diffe'renzierung dier Gewebe und Organe möglich wurde. Beschlossen wird der Unterricht mit der Besprechung des menschlichen Körpers nach Bau und Funktion, dez Anthropologie und der Gesundheitslehre, wobei ständig Fäden zu den Wirbeltieren und auch den Wirbellosen zu knüpfen sind. Hier bietet sich auch Gelegenheit, das Deszendenzproblem zu behandeln, ohne dab es erforderlich oder angebracht wäre, auch auf Fragen wie Darwinismus, Lamarckismus usw. einzugehen, dio dem Hochschulunterricht vorbehalten bleiben mögen.

Gedeihlich kann aber der biologische Unterricht - auch in der bisherigen Beschränkung auf die unteren Klassen - sich nur gestalten, wenn er von Fachbiologen gegeben wird, nicht von ungenügend oder gar nicht vorgebildeten Mathematikern und Physikern im Nebenamt. Ein biologisch gut ausgebil.deter Lehrerstand läBt sich auf die Dauer und in hinreichender Zahl aber nur gewinnen, wenn ihm Gelegenheit geboten wird, sein Fach anch in oberen Klassen vor reiferen Schülern zu vertreten, ein Grund mehr, den Unterricht auch in den oberen Klassen weiterzuführen.

Gelegenheit zur guten Fachausbildung muB der Lehrer schon auf der Hochschule haben. Dem Entwicklungsgange der Zoologie entspiechend stehen im Universitätsbetriebe Morphologie und Physiologie im Vordergrund. Sie werden auch in Zukunft der Angelpunkt der Ausbildung bleiben, nicht allein für den Fachzoologen, sondern auch für den künftigen Lehrer. Daneben soll dem letzteren aber Gelegenheit gegeben werden, sich das nötige Rüstzeug auch für den biologisch-systematischen Unterricht zu verschaffen. Mehr als bisher muß für ent- sprechende Vorlesungen, für Exkursionen und Bestimmungskurse gesorgt werden und mehr als bisher muB dieser Teil der Zoologie im Lehrkörper der Universität durch Ordinariate oder planmäBige Extraordinariate vertreten sein. -

Hertwig sieht also eine Unterbrechung des biologischen Unterrichts in den zwei mittleren Klassen der neunstufigen höheren Lehranstalten vor und weicht in dieser Beziehung von den Hamburger Thesen ab. Wir können aber wohl annehmen, dab er seinen Plan nur als KompromiBvorschlag betrachtet und nicht von der tberzeugung aurgeht, daß die vorgeschlagene Stundenzahl für gedeihliche Behandlung der Biologie völlig ausreiche.

Die vorgeschliagene Teilung des Unterrichtes in einen propädeutischen elementareren Kurs und den darauf aufgebauten tiefer gehenden Kurs der oberen Stufe wird jetzt wohl von allen berufenen Vertretern gutgeheißen, wenn es auch nicht an Stimmen gefehlt hat, die einen durch alle Klassen gleichmäßig im Rahmen der Systematik ausgestalteten Unterricht befürworteten. Nicht die gleiche Einmütigkeit herrscht darüber, wie der gehobene Unterricht in den letzten Klassen zu gestalten sei. Nach Hertwig bild'et das Rückgrat die vergleichende Anatomie und Physiologie, während andere die Okologie in den Mittelpunkt stellen wollten. Die Kommissionsbeschlüsse, die von der Meraner Versammilung gutgeheißen wurden, stellen sich mehr auf den Hertwigschen Standpunkt, tragen aber auch der anderen Auffassung Rechnung. Sie sehen einen anatomisch-physiologischen Unterricht in den beiden obersten Klassen vor, schieben aber ein der Okologie gewidmetes Jahrespensum in der Obersekunda ein.

Hat sich heute die Stellung der Biologie im Unterrichte der höheren Schulen gegen früher auch schon in manchem gebessert, so sind doch weder die Hamburger Thesen, noch die MeranStuttgarter Beschlüsse ganz erfüllt und der Kampf muß noch fortgesetzt werden. Sein Erfolg kann nicht zweifelhaft sein bei der ständig zunehmenden Bedeutung der biologischen Fragen für unser Geistesleben. Die künftigen Generationen, die sich des erreichten Zieles erfreuen können, werden unter den Männern, denen sie Dank schulden, auch Richard Hertwigs nicht vergessen.

\section{Richard Hertwigs Kleines zoologisches Praktikum. \\ Don Otto Koehler, Breslau.}

Die voraufgegangenen Würdigungen der Forscher- und Lehrtätigkeit Richard Hertwigs aus berufenen Federn wenden sich in der Hauptsache an den Zoologen vom Fach. Aber auch zahlreiche Nichitzoologen, besonders Ärzte, Oberlehrer und Landwirte, werden heute in Dankbarkeit ihres 